particle which can be made so massive as to put it beyond observation; the other, of spin two, destroys the theory since it is a ghost particle with a negative norm. As such it violates unitarity, and certainly in particle physics ghosts should frighten one if they can be seen. If this spin-two ghost particle could be decoupled from all other fields then it would have been rendered harmless, though recently it has been realised that such decoupling is very difficult for gravity in the presence of photons or scalar or spin- $-\frac{1}{2}$ particles.

If such is the case there are two ways forward in quantising gravity. One is to start afresh from an alternative beginning to that of Einstein; this is also very difficult since there are almost unavoidable arguments which lead to Einstein's theory of gravity (Deser, J. gen. Rel. Grav., 1, 9-18; 1970). The other alternative is to investigate more carefully the forms of matter for which there is cancellation of the ultraviolet divergences from single loop diagrams. This is a difficult but not impossible problem. Its solution, if there is one, will give a hint as to the form of quantised matter-gravity interaction which is infinity free at all orders; it is to be hoped that there is only one such solution. If there is no such form of theory then gravity will be different indeed from the other forces of nature.

\section{Alpha emission in fast pion reactions}

from $P$. E. Hodgson

MANY researchers in recent years have provided evidence that nucleons in the region of the nuclear surface tend to condense into alpha particles. This naturally affects the cross sections of reactions involving the transfer of a cluster to or from a nucleus, and it is by analysis of data on such reactions that an estimate of the alpha-clustering probability can be obtained.

One of the most direct ways of demonstrating the existence of alpha particles on the nuclear surface is to knock them out with a suitable projectile. Unfortunately the cross sections of these reactions are quite low so that thick targets must be used, and this stops most of the alpha particles from emerging. Instead of trying to detect the alpha particles directly, the residual nuclei after the reaction had taken place were identified from their characteristic gamma-ray spectra. It was then found that a substantial proportion of these nuclei were just the target nucleus less one, two or three alpha particles. This strongly suggested that alpha particles were ejected from the nuclei by

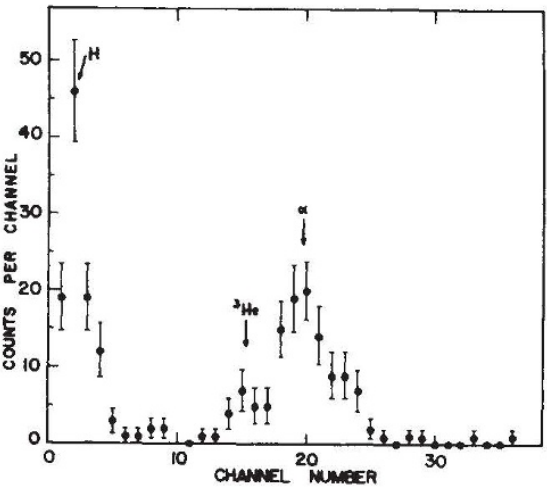

Fig. 1 Particle spectrum showing alpha particles and helions.

the projectiles, though of course the possibility that the nucleons were ejected individually could not be excluded.

This result was found in many experiments using fast and slow kaons and pions as projectiles (Nature, 249, 616 ; 1974).

Some doubt was thrown on the interpretation of these results by an alpha particle knockout process when it was found by statistical model calculations that the observed distribution of final nuclei is what might be expected on purely energetic grounds. It therefore became of great importance to demonstrate the reality of the alpha knockout process by detecting the emitted alpha particles directly.

This has recently been done by a group at Saclay and Tel Aviv (Phys. Rev. Lett., 34, 485; 1975). They bombarded ${ }^{27} \mathrm{Al}$ with $70 \mathrm{MeV}$ negative pions and found a significant cross section for the emission of $\mathrm{He}$ ions, predominantly alpha particles. They were able to overcome the problem of low yield by using the intense electron beam from the Saclay linear accelerator that produces a pion beam of intensity $10^{6}$ pions per second. This made it possible to produce a significant number of alpha particles in a target thin enough for most of them to escape.

The observed particle spectrum at $90^{\circ}$ to the beam is shown in Fig. 1 and has a significant alpha peak and possibly a smaller peak due to helions. The energy spectrum of the He ions in Fig.

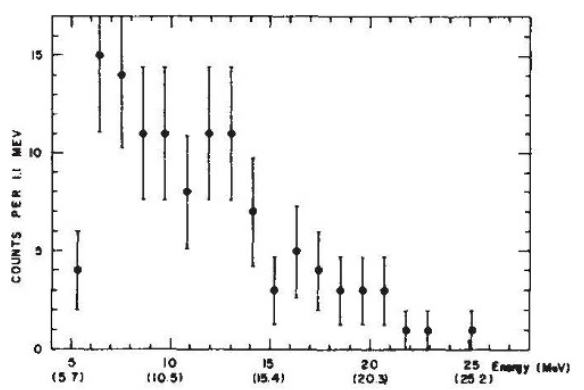

Fig. 2 Energy spectrum of the $\mathrm{He}$ ions emitted from the bombardment of ${ }^{27} \mathrm{Al}$ by $70 \mathrm{MeV}$ negative pions.
2 shows that their intensity has a maximum at about $10 \mathrm{MeV}$, and falls to zero around $25 \mathrm{MeV}$. The differential cross section for apha-particle emission is $6.2 \mathrm{mbarn} \mathrm{sr}^{-1}$ in the energy range 5.5-30 MeV.

Calculations within the framework of a cascade evaporation model indicate that such a yield of alpha particles can be explained by simple evaporation following the excitation of the nucleus by a series of pion-nucleon interactions. Further work is now needed, particularly measurements in the forward direction to see if any of them can be attributed to a direct knockout of preexisting alpha particles on the nuclear surface.

\section{Electric seaweed eggs}

from Julian Lewis

MaNY non-spherical embryos develop from spherical eggs. What then is the origin of their asymmetry? In most cases the fertilised egg, despite its spherical shape, does not have spherical symmetry in its chemical constitution: from the outset there is some local specialisation of the membrane or cortex, or an uneven distribution of material in the cytoplasm. But there is no need in principle for an egg to start life lopsided. It may have at first true spherical symmetry, and lose this through the development of an instability as it matures. Its internal dynamics may be such as to magnify the slightest departure from symmetry into a full-blown polarisation. The egg would thus spontaneously become polarised, but the direction of its polar axis would be subject to control by almost any sort of weak environmental vector.

Jaffe and his colleagues have studied the eggs of the closely related seaweeds Fucus and Pelvetia (Advances in Morphogenesis, 7, 295; 1968). These eggs, about $100 \mu \mathrm{m}$ in diameter, are at first spherical. Within a few hours after fertilisation they glue themselves to the sea bed, and then germinate: they develop a bulge and become pearshaped as the future rhizoid begins to grow out. Practically anything, from a beam of light to a gradient of $p \mathrm{H}$ or a mechanical deformation, can govern the direction in which the rhizoid emerges. This is strongly symptomatic of a symmetry-breaking instability; some other conceivable interpretations can be ruled out experimentally (Jaffe, Science, 123, 1081; 1956).

Thus interest centres on the positivefeedback mechanism in the egg which amplifies and maintains whatever asymmetry happens to arise. Jaffe suggests that a sort of self-electrophoresis 